https://doi.org/10.48009/2_iis_2020_112-122

Issues in Information Systems

Volume 21, Issue 2, pp. 112-122, 2020

\title{
A STUDY OF THE ROLE OF VR IN MENTAL HEALTH BASED ON PUBLIC OPINION
}

\author{
Cian S. Renner, Washburn University, cian.renner@washburn.edu \\ Tyler Cross, Washburn University, tyler.cross@washburn.edu \\ Daniel Corrales, Washburn University, daniel.corrales@washburn.edu \\ Wenying Sun, Washburn University, nan.sun@washburn.edu
}

\begin{abstract}
This research is to investigate who would be open to use Virtual Reality (VR) to help with Mental Health. Is it possible a person's characteristic such as age will affect his/her views on the usefulness of VR to improve mental health? Mental health, both in diagnoses of disorders and treatment of said disorders, is at an all-time high in America. VR has been previously utilized to assess anxieties and phobias such as heights and social anxiety. However, there is very little research of virtual reality mixed with talking therapies such as cognitive-behavioral therapy or counseling. In this research, we collect data through a survey and identify public opinions regarding the advancement of the VR technology and its potential role in mental health.
\end{abstract}

Keywords: Virtual Reality, Public Opinion, Mental Health, Therapy

\section{INTRODUCTION}

In the United States, 1 in 5 adults experience mental illness each year (NIMH, 2019). Out of those with mental illness, only about $43 \%$ receive treatment (Jorm et. al, 2017, p. 1). Some of the top reasons why people don't receive help include not having transportation, concerns about confidentiality, no time during the day, and nowhere to go for service (Susman, 2018). A great way to resolve these problems could be offering virtual reality as a means to get help. Telehealth already provides decent remote access for patients but is not very immersive. A therapy session via video will be a much different experience to that of being in the same room. VR can result in a much more immersive experience, possibly one that can more accurately recreate the feeling of being in the same room, having a private conversation with the therapist.

Virtual reality (VR) has come a long way, especially in the last few years. Looking back on precursors to modern head-mounted display (HMD) technology, the acceleration of VR is apparent. Similarly, while mental health therapy hasn't progressed nearly as much in techniques as VR has in technology, the widespread use of therapy has grown greatly. It is common, now, to hear groups of younger generations talk about their issues with mental health, and their attempts to remedy those issues with therapy. This generation's open and outward view of mental health and subsequent therapy may result in more interest in new therapeutic techniques.

Our goal for this research is to survey the public opinion on application of virtual reality in mental health. We distribute surveys out to the public via social media and email. With our data we hope to answer the following questions:

1. Are people in general open to using virtual reality to cope with mental health?

2. Will a person's characteristics such as age affect his/her opinion (positive or negative) of virtual reality?

The rest of the paper is organized as follows. We first review existing literature on VR, mental health therapy trends, and previous research on applications of VR in mental health therapy. A description of our methodology follows. The results section presents the demographic that participated in the survey, as well as correlations and group comparisons. We then discuss findings, followed by conclusions that can be drawn from this research. 


\section{LITERATURE REVIEW}

In this section, we provide literature review on virtual reality, mental health, and applications of virtual reality to mental health.

\section{Facts about Virtual Reality}

There are several barriers to adopting virtual reality, such as being too expensive, feeling that it can lead to isolation, low interest in games that are available and being afraid of health issues like motion sickness or nausea. According to Statista, 55\% of Americans believe that the biggest barrier is that VR is too expensive (McCarthy, 2019). YouGov found that between 2017 and 2018, the public perception on cost stayed constant, and into the end of 2018 even rose higher (Hiebert, 2019).

Statista collected data on VR ownership in the first quarter of 2017 in the United States and reported the overwhelming majority of the respondents fell into two age groups, 18 to 24 and 25 to 34 . The study found that among all respondents, $37 \%$ of them were VR owners, with 23\% of them falling in the 25 to 34 range, and the other $14 \%$ were between 18 and 24 years old. The study also found that $44 \%$ of the respondents intended to purchase a VR device. Among the respondents intending to purchase a VR device, $27 \%$ of them are between 25 and 34 years old, and $17 \%$ are between 18 and 24 years old (Gough, 2020). Age tends to be the biggest factor in determining who is more likely to use virtual reality.

\section{Facts about Mental Health Therapy}

Treatment for mental health illness has increased in prevalence to "20.1\% in 2001-2003," with evidence pointing towards a continuing increase in the prevalence in recent years (Jorm, et. al, 2017, p. 92). The illnesses in question cover the entire range of known mental illnesses, including anxieties, phobias, depression, and many other disorders. Of those that have a known mental illness, slightly less than half have received mental health service.

American use of mental health services has had a trend of increasing since 1990 (NIMH, 2018). However, this trend is not matched by a lower rate of mental health illness recording. This could be because mental health illnesses are becoming more common in the modern world, or that increased prevalence of mental health therapy has resulted in more common diagnoses of said illnesses. Of course, it could also be a combination of these trends. There has also been evidence that current mental health care standards are not up to par with what they should be. This could also have myriad reasons for occurring. Increased logging of mental health treatment is a necessity to more accurately and usefully track the changing trends in the nation and the world.

It is difficult to focus solely on the prevalence of talking therapy. For one, there are various forms of talking therapy, ranging from cognitive-behavioral therapy (CBT) to counselling to interpersonal therapy (NHS, 2018). The common thread between these types of therapy, and why we will refer to this genre of therapy as "talking therapy", is that they involve and focus mostly on the patient speaking frankly with the health professional, and the health professional responding, attempting to acquire additional information and eventually help the patient reach a more comfortable state of mind (NHS, 2018).

\section{Past Studies Analyzing Virtual Reality Applied to Mental Health Therapy}

Studies applying VR to mental health reported VR was successful at helping patients cope with phobias and PTSD (e.g. Singh and Mishra, 2019, The Franklin Institute, 2020). Notably, VR was "used to recreate the situation that 
triggers the bad dreams or fears thereby making the patient's immune and less reactive to their triggers." (Singh and Mishra, 2019, p. 3738). The authors agree that VR can be applied in many more areas but needs to be explored and made prominent first.

Unfortunately, there was no mention of talking therapies such as cognitive-behavioral therapy or counselling being performed with the aid of VR. This makes it clear this subject has not been adequately explored. Using VR to remotely conduct counselling interviews will be very similar to video and phone interviews, but with an added layer of immersion. However, therapies with more interaction such as the former, cognitive-behavioral therapy, are expected to be far more affected. One of the most common practices in this therapy is letting the patient view their heart rate while doing breathing exercises. VR opens many avenues in visualization and interactivity with the data involved in this practice.

\section{METHODOLOGY}

We hope to gauge public opinion on virtual reality applications in mental health using a survey. The survey is completely online, shared through a recruitment page that details the study's information and has a link to a Google Forms survey. This survey was approved by our institutional review board.

First, questions were proposed and reviewed by the researchers. The survey's point was public opinion, so questions needed to be focused on that. We wished to avoid asking questions about personal experience with therapy but were fine with questions about personal experience with virtual reality. The former was too personal, we decided, and the latter would give valuable data about public familiarity with VR. The survey questions were broken down into sections: Demographics, Virtual Reality, Mental Health Therapy, and Virtual Reality Combined with Mental Health Therapy. Demographics include age, gender, ethnicity, location, level of education, and how strongly the participant identifies with the statement "I am an introvert". The Virtual Reality section includes questions that pertain to how the participant feels about the usefulness of VR. The Mental Health Therapy section seeks to discover how the participant feels about therapy for mental health. All questions pertain to the participant's opinion of the usefulness, efficacy, and availability of mental health therapy. Finally, there is a Combination of Virtual Reality and Mental Health Therapy section that aims to measure public opinion on the concept of applying virtual reality to mental health therapy. The application, specifically, will be both the patient and the therapist using head-mounted displays to enter virtual reality. See appendix A for survey questions.

Data acquisition was performed online with the help of Google Forms. The survey questions were transcribed into a Google Forms file, and the link to this file was shared on Facebook, Twitter, and email. The link was included at the bottom of a recruitment page, outlining what the study's aim is, among other information. The researchers emailed this page to those that showed interest in taking the survey and allowed participants to share the recruitment page with anyone they think would be interested. Google Forms automatically records and displays the survey results, only to the researchers. To analyze the results of the survey, the data is exported to a private Google Sheets document and imported to SPSS Statistics for analysis.

\section{RESULTS}

In this section we report the survey results. We first present the demographic information of the responses. We then report mean, standard error, and correlations of the survey answers. We finally present results on group comparisons.

Our survey received a total of 122 respondents. We received thirty in the 18-21 bracket, twenty-eight in 22-24, eight in 25-29, fifteen in 30-34, four in 35-39, eight in 40-44, five in 45-49, and twenty-four in 50+. 64 respondents were male, 57 were female, and one was in the other category. In the ethnicity section, the majority were Caucasian, with 


\section{Issues in Information Systems \\ Volume 21, Issue 2, pp. 112-122, 2020}

85 respondents. We had 28 Hispanic respondents, 4 African American respondents, 2 Asian respondents, and 3 respondents in the other category. The most common geographic location was the Midwest in the United States, with 104 respondents. In the education section, we received 20 respondents with a high school/GED degree, 25 with a bachelor's, 12 with an associate's, 15 with a graduate degree, and 50 respondents with some college. The respondents that responded with some college are likely current students in our institution. Table 1 is a summary of the demographics of the respondents.

Table 1. Demographics of Respondents

\begin{tabular}{|c|c|c|c|c|c|c|c|c|c|c|c|}
\hline \multicolumn{3}{|c|}{ Gender } & \multicolumn{3}{|c|}{ Age } & \multicolumn{3}{|c|}{ Location } & \multicolumn{3}{|c|}{ Education } \\
\hline Male & 64 & $52.5 \%$ & Under 18 & 0 & $0 \%$ & Midwest US & 104 & $85.2 \%$ & $\begin{array}{l}\begin{array}{l}\text { Some } \\
\text { college }\end{array} \\
\end{array}$ & 50 & $41.0 \%$ \\
\hline Female & 57 & $46.7 \%$ & 18-21 & 30 & $24.6 \%$ & East US & 6 & $4.9 \%$ & Bachelor's & 25 & $20.5 \%$ \\
\hline Other & 1 & $0.8 \%$ & $22-24$ & 28 & $23 \%$ & West US & 5 & $4.1 \%$ & HS/GED & 20 & $16.4 \%$ \\
\hline \multicolumn{3}{|c|}{ Ethnicity } & $25-29$ & 8 & $6.6 \%$ & Outside US & 7 & $5.7 \%$ & Graduate & 15 & $12.3 \%$ \\
\hline Caucasian & 85 & $69.7 \%$ & $30-34$ & 15 & $12.3 \%$ & & & & Associate's & 12 & $9.8 \%$ \\
\hline Hispanic & 28 & $23.0 \%$ & $35-39$ & 4 & $3.3 \%$ & & & & & & \\
\hline $\begin{array}{l}\text { African } \\
\text { American }\end{array}$ & 4 & $3.3 \%$ & $40-44$ & 8 & $6.6 \%$ & & & & & & \\
\hline Asian & 2 & $1.6 \%$ & $45-49$ & 5 & $4.1 \%$ & & & & & & \\
\hline Other & 3 & $2.5 \%$ & $50+$ & 24 & $19.7 \%$ & & & & & & \\
\hline
\end{tabular}

Table 2 presents the mean and standard error of each survey question. The question labels in the table correspond to what is listed in appendix A. In the virtual reality section (V1-V11), the statement the respondents strongly agree with is V5 ( $\mu=4.38)$ - the importance of body language and facial cues. In addition, many respondents do not believe they are proficient at using virtual reality (V11 $\mu=2.30)$. In the therapy section (T1-T6), respondents strongly agree mental health is a necessity for some people (T6 $\mu=4.56$ ), and they do not believe a person have to see therapists face to face to get anything done (T4 $\mu=2.78$ ). In the virtual reality for therapy section, respondents agree moderately with all statements $(\mu>=3.6)$.

Table 2. Basic Statistics

\begin{tabular}{|l|l|l|l|l|l|l|l|}
\hline Q & Mean (SE) & Q & Mean (SE) & Q & Mean (SE) & Q & Mean SE) \\
\hline V1 & $3.73(.11)$ & V7 & $2.90(.15)$ & T1 & $4.31(.07)$ & VT1 & $3.70(.08)$ \\
\hline V2 & $3.06(.10)$ & V8 & $2.40(.11)$ & T2 & $3.59(.10)$ & VT2 & $3.94(.08)$ \\
\hline V3 & $2.75(.11)$ & V9 & $3.54(.10)$ & T3 & $3.58(.10)$ & VT3 & $3.65(.09)$ \\
\hline V4 & $3.60(.09)$ & V10 & $2.30(.12)$ & T4 & $2.78(.09)$ & VT4 & $3.69(.09)$ \\
\hline V5 & $4.38(.07)$ & V11 & $3.59(.09)$ & T5 & $4.20(.08)$ & VT5 & $3.63(.09)$ \\
\hline V6 & \multicolumn{3}{|l|}{$3.11(.12)$} & \multicolumn{7}{|c|}{} & T6 & $4.56(.06)$ & VT6 & $3.76(.08)$ \\
\hline
\end{tabular}

We are also interested in how questions are correlated to each other. We performed a Pearson Correlation Coefficient test to determine which questions had a strong correlation with others. If a Pearson Correlation has a value between $+/-0.5$ and $+/-1$, then it is considered a strong correlation. If the value lies between $+/-0.30$ and $+/-0.49$, then it is considered a medium correlation. If the value lies below $+/-0.29$, then it is considered a small correlation. When reviewing the statistics, some questions stood out. Questions V1 and V2 in our Virtual Reality section had the strongest correlation with a Pearson correlation of 0.664 . Question V1 also had a strong correlation with question V9 with an $\mathrm{r}$ score of 0.627 . Questions T3 and T4 in our Therapy section had an r score of 0.620. In our Virtual Reality Therapy section, questions VT2 and VT5 had an r score of 0.581, while questions VT3 and VT5 had an r score of 0.583. Questions VT4 and V11, which fall under different sections, had an r score of 0.590. Table 3a is a summary of the strongest correlations, and Table 3b displays all correlations. 
Table 3a. Pearson Correlation Coefficient

\begin{tabular}{|l|c|}
\hline Questions & Pearson Correlation \\
\hline V1 \& V2 & 0.664 \\
\hline V1 \& V9 & 0.627 \\
\hline V11 \&VT4 & 0.590 \\
\hline T3 \& T4 & 0.620 \\
\hline VT2 \& VT5 & 0.581 \\
\hline VT3 \& VT5 & 0.583 \\
\hline
\end{tabular}

Table 3b. Correlations

\begin{tabular}{|c|c|c|c|c|c|c|c|c|c|c|c|c|c|c|c|c|c|c|c|c|c|c|c|}
\hline & 1. & & 0. & $v 4$. & & V6. & V7. & vo. & V9. & vio. & V11. & 17. & T2. & 15. & T4. & 15. & 16. & VT1. & VT2. & VT3. & VT4. & VT5. & \\
\hline $\mathrm{v} 1$ & 1 & $.664^{* *}$ & $.478^{* *}$ & $399^{* *}$ & -0.145 & $249^{* *}$ & $.314^{* *}$ & $-.274^{* *}$ & $.627^{* *}$ & $.540^{*+1}$ & 0.126 & 0.001 & -0.101 & -0.120 & -0.111 & 0.096 & -0.004 & $372^{* *}$ & $.476^{* *}$ & $.447^{* *}$ & -0.021 & $.480^{* *}$ & $388^{* *}$ \\
\hline V2 & $.664^{* *}$ & 1 & $.491^{\star \star}$ & $.362^{* *}$ & $-.205^{*}$ & $.329^{* \star}$ & $.186^{*}$ & $-.212^{*}$ & $.548^{* *}$ & $.449^{*+*}$ & 0.007 & .0 .029 & -0.054 & \begin{tabular}{|l|}
-0.070 \\
\end{tabular} & -0.165 & 0.030 & 0.045 & $.299^{* *}$ & $.336^{* *}$ & $.336^{* *}$ & 0.016 & $.349^{* *}$ & $.302^{* *}$ \\
\hline V3 & $.478^{* *}$ & $.491^{* *}$ & 1 & $.499^{* \star}$ & $-.242^{\star \star}$ & $.263^{\star \star *}$ & $.413^{* *}$ & -0.130 & $.349^{* *}$ & $.423^{* *}$ & -0.077 & 0.126 & 0.084 & $-.196^{*}$ & $\mid-277^{* *}$ & 0.111 & 0.063 & $.290^{* \star}$ & $.265^{* *}$ & $.340^{* *}$ & \begin{tabular}{|l|}
-0.036 \\
\end{tabular} & $.244^{* *}$ & $259^{* *}$ \\
\hline V4 & $.399^{\star *}$ & $.362^{\star \star}$ & $.499^{\star \star}$ & 1 & \begin{tabular}{|l|}
-0.137 \\
\end{tabular} & $.349^{* *}$ & $.422^{* \star}$ & -0.093 & $.442^{* *}$ & $.447^{* *}$ & -0.064 & 0.107 & 0.167 & $-.216^{*}$ & \begin{tabular}{|l|}
$-234^{* *}$ \\
\end{tabular} & 0.094 & -0.016 & $.326^{* \star}$ & $.297^{* *}$ & $.276^{* *}$ & $-.182^{*}$ & 0.165 & 0.125 \\
\hline & -0.145 & $-.205^{*}$ & $-.242^{\star *}$ & -0.137 & 1 & $-.290^{* *}$ & $-.189^{*}$ & $.236^{* *}$ & -0.136 & $-.212^{*}$ & 0.013 & $246^{* *}$ & $.293^{* *+}$ & $.243^{* *}$ & $.260^{* *}$ & 0.051 & 0.037 & -0.165 & -0.171 & -0.123 & \begin{tabular}{|c|}
-0.016 \\
\end{tabular} & -0.162 & 0.110 \\
\hline V6 & $.249^{* *}$ & $.329^{* *}$ & $.263^{* *}$ & $.349^{* *}$ & $-.290^{* *}$ & 1 & $.349^{*+}$ & $-.283^{\star *}$ & $.302^{* *}$ & $.303^{*+}$ & 0.154 & 0.013 & \begin{tabular}{|c|}
-0.107 \\
\end{tabular} & -0.088 & \begin{tabular}{|l|}
-0.094 \\
\end{tabular} & -0.109 & 0.029 & 0.157 & $.292^{\star *}$ & $.326^{* *}$ & 0.127 & $.284^{* *}$ & 0.081 \\
\hline V7. & $.314^{* *}$ & $.186^{*}$ & $.413^{*+}$ & $.422^{* *}$ & $-189^{*}$ & $.349^{* *}$ & 1 & -0.173 & $.394^{* * *}$ & $.460^{* *}$ & -0.067 & -0.087 & 0.074 & -0.086 & -0.093 & 0.097 & 0.025 & 0.098 & $.231^{* *}$ & $.241^{* *}$ & -0.126 & 0.059 & 0.088 \\
\hline V8 & $-.274^{* *}$ & $-.212^{*}$ & -0.130 & -0.093 & $.236^{* *}$ & $-.283^{* *}$ & \begin{tabular}{|l|}
-0.173 \\
\end{tabular} & 1 & $83^{* * *}$ & $-.220^{*}$ & -0.061 & 0.125 & 0.125 & $.241^{* *}$ & $.200^{*}$ & 0.057 & 0.170 & $-.267^{* *}$ & $-.359^{* *}$ & $-.213^{*}$ & $\begin{array}{l}-0.056 \\
\end{array}$ & $\mid-345^{* *}$ & -0.040 \\
\hline V9 & $.627^{* *}$ & $.548^{* *}$ & $.349^{* *}$ & $.442^{* *}$ & -0.136 & $.302^{* *}$ & $.394^{* *}$ & $-.283^{* *}$ & 1 & $.457^{*+*}$ & -0.011 & \begin{tabular}{|c|}
-0.059 \\
\end{tabular} & 0.010 & $-.271^{* *}$ & $-.195^{*}$ & $.181^{*}$ & -0.095 & $.427^{* *}$ & $.513^{* *}$ & $.453^{* *}$ & $-.224^{*}$ & $.456^{* *}$ & $.369^{* *}$ \\
\hline V10 & $.540^{* *}$ & $.449^{\star *}$ & $.423^{* *}$ & $.447^{* *}$ & $-.212^{*}$ & $.303^{* *}$ & $.460^{* *}$ & $-.220^{*}$ & $.457^{* *}$ & 1 & 0.021 & 0.053 & 0.099 & -0.081 & \begin{tabular}{|l|}
-0.097 \\
\end{tabular} & 0.061 & 0.020 & $.217^{*}$ & $.229^{*}$ & $.331^{* *}$ & \begin{tabular}{|c|}
-0.076 \\
\end{tabular} & $.241^{* *}$ & $.379^{* *}$ \\
\hline V11 & 0.126 & 0.007 & -0.077 & $\begin{array}{l}-0.064 \\
\end{array}$ & 0.013 & 0.154 & \begin{tabular}{|l|}
-0.067 \\
\end{tabular} & -0.061 & -0.011 & 0.021 & 1 & -0.035 & -0.149 & 0.129 & $.250^{* *}$ & 0.030 & 0.022 & \begin{tabular}{l|l|}
-0.011 \\
\end{tabular} & 0.031 & 0.130 & $.590^{* *}$ & 0.129 & -0.090 \\
\hline $\mathrm{T} 1$. & 0.001 & -0.029 & 0.126 & 0.107 & $246^{* *}$ & 0.013 & \begin{tabular}{|l|}
-0.087 \\
\end{tabular} & 0.125 & -0.059 & 0.053 & .035 & 1 & $.449^{* *}$ & $.192^{*}$ & \begin{tabular}{|l|}
-0.124 \\
\end{tabular} & 0.141 & $.345^{* *}$ & 0.060 & 0.099 & 0.080 & 0.069 & -0.049 & $.295^{* *}$ \\
\hline $\mathrm{T} 2$ & -0.101 & -0.054 & 0.084 & 0.167 & $.293^{* *}$ & \begin{tabular}{|l|}
-0.107 \\
\end{tabular} & 0.074 & 0.125 & 0.010 & 0.099 & -0.149 & $.449^{* *}$ & 1 & $.386^{* *}$ & 0.119 & $.266^{* *}$ & 0.141 & -0.075 & -0.008 & -0.040 & \begin{tabular}{|l|}
-0.147 \\
\end{tabular} & -0.158 & $.201^{*}$ \\
\hline T3. & -0.120 & -0.070 & $-1.196^{*}$ & $-.216^{*}$ & $.243^{* *}$ & \begin{tabular}{|l|}
-0.088 \\
\end{tabular} & $\begin{array}{l}-0.086 \\
\end{array}$ & $241^{* *}$ & $-.271^{\text {***}}$ & -0.081 & 0.129 & $.192^{*}$ & $.386^{* *}$ & 1 & $.620^{* *}$ & -0.039 & 0.062 & $-.274^{* *}$ & \begin{tabular}{|l|}
$.319^{* *}$ \\
\end{tabular} & $\mid-.252^{* *}$ & 0.040 & \begin{tabular}{|l|}
$-.279^{* *}$ \\
\end{tabular} & -0.062 \\
\hline $\mathrm{T} 4$ & -0.111 & -0.165 & $-.277^{\star \star}$ & $-.234^{* *}$ & $260^{* *}$ & -0.094 & -0.093 & $.200^{*}$ & $-.195^{*}$ & -0.097 & $.250^{* *}$ & -0.124 & 0.119 & $.620^{* *}$ & 1 & 0.056 & -0.009 & $-.265^{\star \star \star}$ & $\mid-.245^{\star *}$ & $-.182^{*}$ & 0.116 & -0.152 & -0.153 \\
\hline T5 & 0.096 & 0.030 & 0.111 & 0.094 & 0.051 & -0.109 & 0.097 & 0.057 & $.181^{*}$ & 0.061 & 0.030 & 0.141 & $.266^{* *}$ & -0.039 & 0.056 & 1 & $.272^{* *}$ & -0.035 & 0.080 & 0.050 & 0.080 & 0.029 & 0.010 \\
\hline T6. & -0.004 & 0.045 & 0.063 & -0.016 & \begin{tabular}{l|l|}
0.037 \\
\end{tabular} & 0.029 & 0.025 & 0.170 & -0.095 & 0.020 & 0.022 & $.345^{* *}$ & 0.141 & 0.062 & \begin{tabular}{|l|}
-0.009 \\
\end{tabular} & $.272^{* *}$ & 1 & -0.082 & -0.015 & -0.032 & 0.155 & $-.179^{*}$ & -0.014 \\
\hline VT1 & $.372^{* *}$ & $299^{* *}$ & $.290^{* *}$ & $.326^{* *}$ & \begin{tabular}{|l|}
-0.165 \\
\end{tabular} & 0.157 & 0.098 & $-.267^{* *}$ & $.427^{* *}$ & $.217^{*}$ & -0.011 & 0.060 & -0.075 & $-.274^{* *}$ & $-.265^{* *}$ & -0.035 & -0.082 & 1 & $.467^{* *}$ & $.527^{* *}$ & -0.094 & $.552^{* *}$ & $.323^{*+}$ \\
\hline VT2. & $.476^{\star * *}$ & $.336^{\star *}$ & $.265^{\star \star *}$ & $.297^{\star \star *}$ & \begin{tabular}{|l|}
-0.171 \\
\end{tabular} & $292^{* *}$ & $.231^{* *}$ & $-.359^{* *}$ & $.513^{* * *}$ & $.229^{*}$ & 0.031 & 0.099 & -0.008 & $-.319^{* *}$ & $-.245^{* *}$ & 0.080 & -0.015 & $.467^{* *+}$ & 1 & $.529^{* *}$ & 0.008 & $.581^{* *}$ & $.333^{*+}$ \\
\hline VT3 & $.447^{\star \star}$ & $.336^{* *}$ & $.340^{\star \star}$ & $.276^{\star \star *}$ & \begin{tabular}{|l|}
-0.123 \\
\end{tabular} & $.326^{* \star}$ & $.241^{* *}$ & $-.213^{*}$ & $.453^{* *}$ & $.331^{*+}$ & 0.130 & 0.080 & -0.040 & $-.252^{* *}$ & $-.182^{\star}$ & 0.050 & -0.032 & $.527^{* *}$ & $.529^{* *}$ & 1 & -0.065 & $.583^{*+}$ & $.280^{* *}$ \\
\hline VT4. & -0.021 & 0.016 & -0.036 & $-.182^{*}$ & \begin{tabular}{|l|}
-0.016 \\
\end{tabular} & 0.127 & -0.126 & -0.056 & $-.224^{*}$ & -0.076 & $.590^{* *}$ & 0.069 & -0.147 & 0.040 & 0.116 & 0.080 & 0.155 & -0.094 & 0.008 & .065 & 1 & -0.009 & -0.072 \\
\hline VT5. & $.480^{* *}$ & $.349^{* *}$ & $.244^{* *}$ & 0.165 & \begin{tabular}{|c|}
-0.162 \\
\end{tabular} & $.284^{* *}$ & 0.059 & $-.345^{* *}$ & $.456^{* * *}$ & $.241^{* *}$ & 0.129 & .049 & -0.158 & $-.279^{* *}$ & $\begin{array}{l}-0.152 \\
\end{array}$ & 0.029 & $\mid-179^{*}$ & $.552^{* *}$ & $.581^{* *}$ & $.583^{* *}$ & -0.009 & 1 & $.278^{* *}$ \\
\hline VT6. & $.388^{* *}$ & $02^{* *}$ & $.259^{* \star}$ & 0.125 & 0.110 & 081 & .088 & .040 & $.369^{* *}$ & $99^{* *}$ & 090 & $.295^{* *}$ & $.201^{*}$ & .062 & \begin{tabular}{|l|}
-0.153 \\
\end{tabular} & 0.010 & -0.014 & $323^{* *}$ & $33^{* *}$ & $280^{* *}$ & .072 & $.278^{* *}$ & 1 \\
\hline
\end{tabular}

We would like to know whether a person's characteristics such as age, race, and gender influenced the answers. We first looked at age. We settled on two groups, Under 29 and 30+. We debated splitting up the 30+ section into two groups, 30-49 and 50+. We identified no statistical difference between those two groups, so we decided to leave it as $30+$. We used an independent sample t-test to compare our survey questions between two age groups, under 29 and $30+$.

Tables 4, 5, 6 present a summary of the mean, standard error mean, mean difference, t-value, and p-value of each of the questions split into the two age groups. Table 4 shows there are statistically significant differences between the two age groups on most virtual reality questions (p-value $<=0.02$ ), with three exceptions: V5, V8, V11. In the Therapy section, as demonstrated in Table 5 , there is no difference between the two age groups on any of the questions (pvalue $>=0.23$ ). In the Virtual Reality for Therapy section, the mean for every question was well above 3, which indicates that the age groups, on average, agree with the question. Except for two questions (VT1 and VT4, p-value $>=0.14$ ) the data shows statistically significant differences between the two age groups on all the other questions (pvalue $<=0.04$ ). 


\section{Issues in Information Systems}

Volume 21, Issue 2, pp. 112-122, 2020

Table 4. Virtual Reality Age Groups Mean Comparison

\begin{tabular}{|c|c|c|c|c|c|c|c|c|c|c|c|}
\hline \multirow[t]{2}{*}{$\mathbf{Q}$} & Age 1 & Age 2 & Mean & t-value & \multirow[t]{2}{*}{ p-value } & \multirow[t]{2}{*}{$\mathbf{Q}$} & Age 1 & Age 2 & \multirow{2}{*}{$\begin{array}{l}\text { Mean } \\
\text { Diff. }\end{array}$} & \multirow[t]{2}{*}{ t-value } & \multirow[t]{2}{*}{ p-value } \\
\hline & Mean & Mean & Diff. & & & & Mean & Mean & & & \\
\hline V1 & 4.28 & 3.07 & 1.21 & 6.402 & 0.001 & V2 & 3.40 & 2.64 & .76 & 3.996 & 0.001 \\
\hline V3 & 2.93 & 2.54 & 0.39 & 1.762 & 0.081 & V4 & 3.85 & 3.30 & 0.55 & 2.969 & 0.004 \\
\hline V5 & 4.37 & 4.39 & -0.02 & -0.170 & 0.865 & V6 & 3.35 & 2.82 & 0.53 & 2.300 & 0.023 \\
\hline V7 & 3.13 & 2.61 & 0.52 & 1.784 & 0.077 & V8 & 2.29 & 2.54 & -0.25 & -1.046 & 0.298 \\
\hline V9 & 3.87 & 3.14 & 0.73 & 3.741 & 0.001 & V10 & 2.72 & 1.79 & 0.93 & 4.349 & 0.001 \\
\hline V11 & 3.65 & 3.52 & 0.13 & 0.721 & 0.472 & & & & & & \\
\hline
\end{tabular}

*Age 1=Under 29, Age 2=30 and over

Table 5. Therapy Age Groups Mean Comparison

\begin{tabular}{|l|l|l|l|l|l|l|l|l|l|l|l|}
\hline Q & Age 1 & Age 2 & Mean & t-value & p-value & Q & Age 1 & Age 2 & $\begin{array}{l}\text { Mean } \\
\text { Dif-value }\end{array}$ & p-value \\
\cline { 2 - 8 } & Mean & Mean & Diff. & & & & Mean & Mean & & \\
\hline D1 & 4.26 & 4.36 & -0.10 & -0.634 & 0.527 & T2 & 3.56 & 3.63 & -0.07 & -0.330 & 0.742 \\
\hline T3 & 3.49 & 3.70 & -0.21 & -1.121 & 0.265 & T4 & 2.69 & 2.89 & -0.20 & -1.078 & 0.283 \\
\hline T5 & 4.24 & 4.16 & 0.08 & 0.451 & 0.653 & T6 & 3.35 & 2.82 & 0.53 & 1.198 & 0.233 \\
\hline
\end{tabular}

Table 6. Virtual Reality for Therapy Age Groups Mean Comparison

\begin{tabular}{|l|l|l|l|l|l|l|l|l|l|l|l|}
\hline Q & Age 1 & Age 2 & Mean & t-value & p-value & Q & Age 1 & Age 2 & $\begin{array}{l}\text { Mean } \\
\text { Diff. }\end{array}$ & t-value & p-value \\
\cline { 2 - 9 } & Mean & Mean & Diff. & & & & Mean & Mean & \\
\hline VT1 & 3.81 & 3.57 & 0.24 & 1.473 & 0.143 & VT2 & 4.09 & 3.77 & 0.32 & 2.155 & 0.033 \\
\hline VT3 & 3.87 & 3.39 & 0.48 & 2.610 & 0.010 & VT4 & 3.75 & 3.63 & 0.12 & 0.698 & 0.487 \\
\hline VT5 & 3.84 & 3.38 & 0.46 & 2.253 & 0.013 & VT6 & 3.91 & 3.57 & 0.34 & 2.045 & 0.043 \\
\hline
\end{tabular}

The next few tables showcase differences between the two largest groups in our ethnicity demographic: Caucasian and Minorities. With few individual respondents of African American, Hispanic, Asian, and Other, we decided to group them together to see if any large differences arose.

Table 7 shows data in the virtual reality section. On four questions (V5, V6, V8, V11) there are significant differences between the two race categories (p-value $<=0.005$ ). Table 8 presents mean comparisons on therapy questions. Only on two questions (T2, T3) the mean differences are statistically significant (p-value $<=0.03$ ). In the virtual reality for therapy (Table 9) section the mean differences on all questions are statistically significant (p-value $<=0.03$ ).

Table 7. Virtual Reality Ethnicity Groups Mean Comparison

\begin{tabular}{|l|l|l|l|l|l|l|l|l|l|l|l|}
\hline \multirow{2}{*}{ Q } & C & M & Mean & t-value & p-value & $\mathbf{Q}$ & $\mathbf{C}$ & $\mathbf{M}$ & $\begin{array}{l}\text { Mean } \\
\text { Diff. }\end{array}$ & t-value & p-value \\
\cline { 2 - 9 } & Mean & Mean & Diff. & & & & Mean & Mean & \\
\hline V1 & 3.65 & 3.92 & -0.27 & -1.153 & 0.251 & V2 & 3.02 & 3.13 & -0.11 & -0.525 & 0.601 \\
\hline V3 & 2.80 & 2.63 & 0.17 & 0.683 & 0.497 & V4 & 3.63 & 3.55 & 0.08 & 0.394 & 0.695 \\
\hline V5 & 4.57 & 3.95 & 0.62 & 4.189 & 0.001 & V6 & 2.90 & 3.61 & -0.71 & -2.838 & 0.005 \\
\hline V7 & 3.01 & 2.63 & 0.38 & 1.211 & 0.230 & V8 & 2.66 & 1.82 & 0.84 & 3.571 & 0.001 \\
\hline V9 & 3.49 & 3.66 & -0.17 & -0.77 & 0.443 & V10 & 2.30 & 2.30 & 0.00 & 0.052 & 0.959 \\
\hline V11 & 3.40 & 4.03 & -0.63 & -3.264 & 0.002 & \multicolumn{10}{|c|}{} \\
\hline
\end{tabular}

**C=Caucasian, $\mathrm{M}=$ Minorities 
Table 8. Therapy Ethnicity Group Mean Comparison

\begin{tabular}{|c|c|c|c|c|c|c|c|c|c|c|c|}
\hline \multirow[t]{2}{*}{$\mathbf{Q}$} & $\mathrm{C}$ & $\mathbf{M}$ & \multirow{2}{*}{$\begin{array}{l}\text { Mean } \\
\text { Diff. }\end{array}$} & \multirow[t]{2}{*}{ t-value } & \multirow[t]{2}{*}{ p-value } & \multirow[t]{2}{*}{$\mathbf{Q}$} & \multirow{2}{*}{$\begin{array}{l}\text { C } \\
\text { Mean }\end{array}$} & \multirow{2}{*}{$\begin{array}{l}\text { M } \\
\text { Mean }\end{array}$} & \multirow{2}{*}{$\begin{array}{l}\text { Mean } \\
\text { Diff. }\end{array}$} & \multirow[t]{2}{*}{ t-value } & \multirow[t]{2}{*}{ p-value } \\
\hline & Mean & Mean & & & & & & & & & \\
\hline T1 & 4.37 & 4.16 & 0.21 & 1.502 & 0.137 & $\mathrm{~T} 2$ & 3.91 & 2.87 & 1.04 & 5.446 & 0.001 \\
\hline T3 & 3.71 & 3.29 & 0.42 & 2.196 & 0.031 & $\mathrm{~T} 4$ & 2.81 & 2.71 & 0.10 & 0.557 & 0.579 \\
\hline T5 & 4.24 & 4.11 & 0.13 & 0.743 & 0.460 & T6 & 4.60 & 4.47 & 0.13 & 1.026 & 0.308 \\
\hline
\end{tabular}

Table 9. Virtual Reality for Therapy Ethnicity Group Mean Comparison

\begin{tabular}{|c|c|c|c|c|c|c|c|c|c|c|c|}
\hline \multirow[t]{2}{*}{$\mathbf{Q}$} & C & $\mathbf{M}$ & \multirow{2}{*}{$\begin{array}{l}\text { Mean } \\
\text { Diff. }\end{array}$} & \multirow[t]{2}{*}{ t-value } & \multirow[t]{2}{*}{ p-value } & \multirow[t]{2}{*}{$\mathbf{Q}$} & $\mathrm{C}$ & & \multirow{2}{*}{$\begin{array}{l}\text { Mean } \\
\text { Diff. }\end{array}$} & \multirow[t]{2}{*}{ t-value } & \multirow[t]{2}{*}{ p-value } \\
\hline & Mean & Mean & & & & & Mean & & & & \\
\hline VT1 & 3.81 & 3.57 & 0.24 & 2.949 & 0.004 & VT2 & 4.09 & 3.77 & 0.32 & 2.949 & 0.004 \\
\hline VT3 & 3.87 & 3.39 & 0.48 & 2.94 & 0.004 & VT4 & 3.75 & 3.63 & 0.12 & 3.428 & 0.001 \\
\hline VT5 & 3.84 & 3.38 & 0.46 & 3.724 & 0.001 & VT6 & 3.91 & 3.57 & 0.34 & 2.184 & 0.032 \\
\hline
\end{tabular}

Group comparisons were performed on Gender as well, but we found no statistically significant differences.

At the end of the survey we added an option for comments. This provided respondents an opportunity to give qualitative feedback on their opinions of VR mental health. 15 respondents provided comments. Five comments were removed due to irrelevance to our subject or for being too short for meaningful analysis. The comments are shown below.

Table 10. Comments

\begin{tabular}{|c|l|}
\hline$\#$ & \multicolumn{1}{c|}{ Comment } \\
\hline 1 & $\begin{array}{l}\text { "Some people may feel more comfortable expressing their feeling not being face to face with another } \\
\text { person" }\end{array}$ \\
\hline 2 & $\begin{array}{l}\text { "What an interesting concept! I'd never thought about it...but very intriguing! Question of costs is } \\
\text { certainly raised but I imagine that we can find a way to get around that! Virtual reality I assume relies } \\
\text { on high speed internet...which raises the question of rural America which already suffers from a lack } \\
\text { of good affordable highspeed internet as well as good quality accessible mental health care! That } \\
\text { could probably be a whole other discussion in itself!" }\end{array}$ \\
\hline 3 & $\begin{array}{l}\text { "I think this could be a great idea as long as you are able to interact with a real therapist in real time. } \\
\text { I don't think this would work if using a programmed avatar/computer agent." }\end{array}$ \\
\hline 4 & $\begin{array}{l}\text { "I think it has possibilities, but a lot of work would need to be done to make it accessible, and I think } \\
\text { you really need to be very cautious of ethical practice. Not sure how much research has been done on } \\
\text { the effectiveness, either. There is some evidence that use of avatar in therapy is effective with some } \\
\text { populations and disorders so I imagine virtual reality may have its place in therapy as well.” }\end{array}$ \\
\hline 5 & $\begin{array}{l}\text { "Disclosure: I am a licensed therapist with 15 years of experience. COVID has opened up a lot of new } \\
\text { telehealth options (temporarily). Which have posed some challenges for accessibility, infection } \\
\text { control and insurance coverage. (I work with older adults and we just started telehealth). I'm interested } \\
\text { in your work, seems like a lot of opportunity." }\end{array}$ \\
\hline 6 & $\begin{array}{l}\text { "I see a mental health therapist once every two weeks. I feel like having access to a therapist through } \\
\text { virtual reality might be more helpful for me. I can meet once a week from my home and not have to } \\
\text { figure out ways to place something so important in my schedule.” }\end{array}$ \\
\hline
\end{tabular}


Table 10. Comments

\begin{tabular}{|c|l|}
\hline$\#$ & \multicolumn{1}{c|}{ Comment } \\
\hline 7 & $\begin{array}{l}\text { "This is actually really cool and something I haven't thought of before! I think VR is expensive, but } \\
\text { so is therapy, so I think people who are more comfortable with technology and less comfortable in a } \\
\text { face to face conversation could benefit from this greatly. Maybe not for everyone, but a good way to } \\
\text { go about it for others!" }\end{array}$ \\
\hline 8 & $\begin{array}{l}\text { "I think it could help a lot but wonder how many people that it could benefit have access to virtually } \\
\text { reality or would go get the equipment necessary to is virtual reality in this context if they don't already } \\
\text { have the equipment necessary" }\end{array}$ \\
\hline 9 & $\begin{array}{l}\text { "I feel like that if the therapist has VR technology it would help them communicate with the patient } \\
\text { as the therapist sees how the patient reacts differently in person to the therapist and over internet with } \\
\text { friends and it would pose another way for the therapy to help the fears as if they were afraid of } \\
\text { something it would get them used to it without hurting them, since it is all in the headset and not in } \\
\text { real life, it may take longer, but it is something that hasn't been tried and could have major success" }\end{array}$ \\
\hline 10 & $\begin{array}{l}\text { "It is not something I have considered before, but I can see using a virtual avatar to be helpful for } \\
\text { some people so they have some control over how they are seen or represented. I think a lot of our day- } \\
\text { to-day mental battles are about how we are perceived by others (social anxiety), and having complete } \\
\text { control of your appearance can be powerful regardless of the setting." }\end{array}$ \\
\hline
\end{tabular}

\section{DISCUSSION}

Analysis of the survey results shows that overall, respondents agreed with the questions about VR, Therapy, and Virtual Reality Therapy. Questions of note in the VR section are V10, which had a score very close to disagree, and V5, which had the highest agree score in this section. V10 gauged proficiency with VR technology, so this result tells us that people, in general, do not feel proficient with the technology. This makes sense, as the technology is currently expensive in price and space needed. V5 gauged how important facial cues are to understand a person. The high score for this question is expected. It can be hard to tell a person's intent without seeing their facial expressions. The Therapy section had even higher average scores than the VR section. Notably, the final question, T6, gauged how necessary people feel mental health therapy is to some. The respondents obviously feel that therapy is a necessity for some people, with this question scoring closer to strongly agree than agree. T4, which stated that an individual would need to see their therapist face-to-face to get anything done, got a score slightly below neutral, indicating slight disagreement. This increases the viability of VR applied to therapy, since if people feel face-to-face meetings are not necessarily needed, VR can be used with less loss of therapeutic efficacy. The VR Therapy questions all leaned towards agree. The lowest score, VT5 was still closer to agree than neutral. This question asked if therapists investing in VR technology was a good idea, so apparently the respondents agree. VT2 was the highest scoring question, and it gauged if the respondent thinks VR could expand the reach of therapists. The respondents agree, showing understanding of the technology's uses.

A significant difference in the Age vs VR Therapy section was noted during analysis. Participants under the age of 30 have a higher belief in mental health therapists investing in virtual reality compared to participants with age of 30 or above. The young respondents also believe that it would be easier for people to talk to a mental health therapist if they were in virtual reality. These point to our original assertion that younger people under 30 are more likely to try VR. Most of it has to do with the age in which we were all born. Technology has made significant advancements in the last thirty years and thus has helped the people growing up in this era to be more open to new technology.

We found that participants under the age of 30 seemed more interested in and open to virtual reality technology used in the world of mental health therapy. This most likely has a multifaceted reason for occurring. Younger individuals have been around more advanced technology for, proportionally, more of their lives than older individuals. Individuals that have had advanced technology surrounding them from a young age will naturally be more comfortable with said 
technology, whereas older individuals, whose worlds have been quickly encroached by the rapid acceleration of technological advancement, are more likely to be out of the loop. Reduced knowledge of technology is expected to increase the likelihood of avoidance or rejection of the technology. There was very little difference between our established age groups in their opinion of mental health therapy. This was surprising, as we expected the younger group to be more open, and the older group to be less open. Instead, opinions seem to be about the same. It seems, then, that increased awareness about the importance of mental health therapy has spread evenly in regard to age.

It was found that Caucasian respondents differed somewhat from Minorities respondents. Notably in the VR section, V5, V6, V8, and V11's answers differed. V6 measured how much respondents talked to people online compared to real life. Our Caucasian demographic was found to disagree slightly with the question, while our Minorities demographic agreed slightly. This shows our respondents that are in the Minorities section communicate online more than our Caucasian respondents. V8 gauged how motion sick respondents think they would feel in VR. Respondents in the Minorities section disagreed with the question, while Caucasian respondents disagreed as well, but not as heavily. This may show that Caucasian respondents feel more comfortable with VR technology, as they feel less likely to experience motion sickness.

The comments left on our survey bring up great points. First, someone that sees a therapist often will gain more from VR-applied mental health. Being remote, they can just put on a head-mounted display and save the repeated trips to the therapist's office. The second comment points out that some people will feel more comfortable avoiding face-toface expression. It can be difficult for those with social anxiety to build up the courage to go see a therapist, so telehealth solutions may make it easier. VR-assisted therapy may provide an essential in-room experience that audioonly telehealth cannot replicate, as well. Comparisons of how the patient will react to similar situations online and inperson may also yield valuable information for the therapist to share with the patient. Anonymity has become much more common for people to experience with the advent of online communication. Said anonymity may make people act differently than they would in real life and create habits that need to be analyzed by professionals.

The COVID-19 pandemic has resulted in more people staying at home, with virtual means being a more common way to connect with others. We collected data just a few weeks after stay-at-home advisories were set in place in many locations in the United States. The respondents' opinions are likely to have changed due to these conditions. Telehealth has been a topic of interest for mental health providers, as it's the only way they can keep appointments running during this. Like office jobs, individuals may find that telehealth solutions work better for them than in-person. This could possibly result in more adoption of virtual reality for situations that prefer a presence in the room.

\section{CONCLUSIONS, LIMITATION, FUTURE RESEARCH}

This research aimed to answer two questions: Will a person's characteristics such as age, race affect one's opinion of virtual reality? And are people in general open to using virtual reality to cope with mental health? We conducted a survey that was distributed through social media. From analysis of the survey data, we identify that people generally believe body language and facial cues are important to understanding someone, mental health therapy is a necessity for some people, transportation is a barrier for some people to see a therapist, and virtual reality could expand the reach of mental health therapy but is too expensive. We also noticed that people of a younger age were, on average, more open to using virtual reality in mental health.

Our research has several limitations. The most notable is the small sample size. We had 122 respondents. At this small scale, it is difficult to extrapolate the results to a larger view of public opinion. The Ethnicity and Location turnout was affected by this as well. Most respondents identify as Caucasian and are from the Midwest US. While the Midwest US is the option that covers the most land, the East and West US have very different cultures and population densities. 
This means our study mostly looked at Midwest US Caucasian opinions on virtual reality applications in mental health therapy. Online distribution of the survey was mostly effective, but some on-foot distribution could have helped as well. Unfortunately, the COVID-19 pandemic interrupted on-foot survey distribution, forcing us to move to solely online distribution.

Additional survey research will lead to a clearer view of public opinion, as well, looking at the same topic as ours. Another stream of research will be for researchers to conduct experiments analyzing how effective talking therapies are in person and in virtual reality. This would answer the question if using virtual reality in mental health is effective.

\section{REFERENCES}

Gough, C. (2020). VR and AR ownership and purchase intent among U.S. consumers 2017, by age, Statista [online], - VR and AR ownership in the US by age 2017.

Hiebert, P. (2020). After a Brief Pause, VR Adoption Grows to 11\% Among All US Students, YouGov [online], After a brief pause, VR adoption grows to $11 \%$ among all US adults.

History of Virtual Reality (2020), Virtual Reality Society [online], History Of Virtual Reality.

Jorm, A. F., Patten, S. B., Brugha, T. S. \& Mojtabai, R. (2017), Has increased provision of treatment reduced the prevalence of common mental disorders? Review of the evidence from four countries. World Psychiatry, 16: 90-99. doi:10.1002/wps.20388.

McCarthy, N. (2019) Familiarity with VR Increases but Cost Remains a Hurdle, Statista [online], Infographic: Familiarity With VR Increases But Cost Remains A Hurdle.

Mental Illness (2020), National Institute of Mental Health [online], Mental Illness.

Singh, M. \& Mishra, K. D. (2019). Applications of Virtual Reality in Mental Health, International Journal of Recent Technology and Engineering [online], International Journal of Recent Technology and Engineering

Susman, D. (2018). The Top Reasons Why People Don’t Receive Needed Mental Health Services, davidsusman.com [online], The Top Reasons Why People Don’t Receive Needed Mental Health Services

The Science of Virtual Reality (2020). The Franklin Institute [online], The Science of Virtual Reality.

Types of Talking Therapies (2020), National Health Service [online], Types of talking therapies.

\section{APPENDIX A - SURVEY INSTRUMENT}

Virtual Reality 1 (Strongly disagree) to 5 (Strongly agree)

V1. I have interest in using virtual reality technology.

V2. I believe virtual reality would create a real-life like experience for me.

V3. I believe virtual experiences are just as valuable as real life experiences.

V4. I can relate to someone when talking to them online.

V5. Body language and facial cues are important to understanding someone.

V6. I talk with people online at least as much as I do in real life. 
V7. I have friends I only know online.

V8. I believe virtual reality would make me motion sick.

V9. I would be comfortable meeting friends in virtual reality.

V10. I believe I am proficient at using virtual reality.

V11. I believe virtual reality technology is too expensive.

Therapy 1 (Strongly disagree) to 5 (Strongly agree)

T1. I believe talking to a mental health therapist can be helpful in general.

T2. I believe the room a mental health therapist conducts therapy sessions in matters.

T3. I believe that seeing a mental health therapist in real life is better than over the phone or video.

T4. I believe a person would need to see their mental health therapist face-to-face to get anything done.

T5. Transportation is a barrier for some people to see a therapist.

T6. I see mental health therapy as a necessity for some people.

Virtual Reality with Therapy for Mental Health 1 (Strongly disagree) to 5 (Strongly agree)

VT1. I believe virtual reality technology is advanced enough to be of use in therapy for mental health.

VT2. I believe virtual reality will expand the reach of mental health therapy.

VT3. I believe it would be easier for people to talk to a mental health therapist if they were in virtual reality.

VT4. I believe virtual reality for mental health would cost too much for the patient.

VT5. I think it is a good idea for mental health therapists to invest in virtual reality.

VT6. I believe virtual reality may help people that suffer from phobias, such as heights or spiders. 